\title{
Correlation between Exchange Bias and Pinned Interfacial Spins
}

\author{
H. Ohldag, ${ }^{1,2,3, *}$ A. Scholl, ${ }^{2}$ F. Nolting, ${ }^{4}$ E. Arenholz, ${ }^{2}$ S. Maat,${ }^{5}$ A. T. Young, ${ }^{2}$ M. Carey, ${ }^{5}$ and J. Stöhr ${ }^{1}$ \\ ${ }^{1}$ Stanford Synchrotron Radiation Laboratory, P.O. Box 20450, Stanford CA 94309, USA \\ ${ }^{2}$ Advanced Light Source, One Cyclotron Road, Lawrence Berkeley National Laboratory, Berkeley CA 94720, USA \\ ${ }^{3}$ Institut für Angewandte Physik, Universität Düsseldorf, 40225 Düsseldorf, Germany \\ ${ }^{4}$ Swiss Light Source, Paul Scherrer Institut, CH-5232 Villigen PSI, Switzerland \\ ${ }^{5}$ IBM Research Division, Almaden Research Center, 650 Harry Road, San Jose CA 95120, USA
}

\begin{abstract}
Using x-ray magnetic circular dichroism we have detected the very interfacial spins that are responsible for the horizontal loop shift in three different exchange bias sandwiches, chosen because of their potential for device applications. The "pinned" uncompensated interfacial spins constitute only a fraction of a monolayer and do not rotate in an external magnetic field since they are tightly locked to the antiferromagnetic lattice. A simple extension of the Meiklejohn and Bean model is proposed to quantitatively account for the exchange bias fields in the three studied systems from the experimentally determined number of pinned moments and their sizes.
\end{abstract}

Many of today's advanced magnetic devices, such as spin-valves for magnetic recording read heads [1], rely on an effect called exchange bias where the magnetization of a ferromagnetic layer is pinned into a well-defined reference direction by an antiferromagnet such that its hysteresis loop exhibits a horizontal shift $[2,3]$. Extensive research has led to the notion that the exchange bias effect must originate from uncompensated interfacial spins that are anchored in the antiferromagnet (i.e. are pinned) and do not follow the external field (for a review see $[4,5])$. Today, the detection of the pinned spins, the determination of their origin and size, and their quantitative link to the size of the bias effect remain forefront research problems.

Uncompensated spins associated with antiferromagnets or their interfaces have been observed in several systems, such as $\mathrm{CoO} / \mathrm{MgO}[6]$ using SQUID or $\mathrm{Co} / \mathrm{FeMn}$ [7], Co/IrMn [8] and $\mathrm{Co} / \mathrm{NiO}$ [9] using XMCD. In particular, the study of $\mathrm{Co} / \mathrm{NiO}$ showed that the existence of uncompensated interfacial spins is insufficient for exchange bias since they may more strongly couple to and rotate with the ferromagnet, yielding no bias. Experimental evidence for pinned spins has only been reported for single-crystal-like $\mathrm{Fe} / \mathrm{FeF} \mathrm{F}_{2}$ and $\mathrm{Fe} / \mathrm{MnF}_{2}$ where a perpendicular coupling between the spin axes of the ferromagnet (FM) and antiferromagnet (AFM) has been suggested [10]. Hysteresis loops obtained from these samples also exhibited a vertical loop shift indicating the presence of pinned spins in the sample. However the exact location of the pinned spins could not be specified. Hence a quantitative correlation between the experimentally determined pinned magnetization and the macroscopic exchange bias field, crucial to the microscopic understanding of exchange bias, has not been achieved so far.

Here we present results for three polycrystalline exchange bias sandwiches, $\mathrm{NiO} / \mathrm{Co}$, IrMn/Co and $\mathrm{PtMn} / \mathrm{Co}_{90} \mathrm{Fe}_{10}$, chosen for their technological relevance and prototypical behavior. We used high sensitivity $\mathrm{x}$ ray magnetic circular dichroism (XMCD) spectroscopy in total electron yield (TEY) detection to identify un- compensated Ni or Mn spins, located at the respective AFM-FM interfaces [9]. The TEY method offers sensitivity to the interfacial region because of the limited $1 / e$ probing depth, as explained in detail previously $[9,11]$. Significant changes in the XAS line shape are detected if chemical or magnetic properties of the interfacial layer deviate from bulk properties. By measurement of the $\mathrm{Ni}$ or Mn XMCD hysteresis loops we show that only a small fraction of the interfacial spins, about $4 \%$ of a monolayer (ML), is tightly pinned to the AFM and does not rotate in an external field. The size of the pinned interfacial magnetization is found to be quantitatively correlated to the macroscopic magnetic exchange bias field, using a modified simple model, originally suggested by Meiklejohn and Bean $[2,3]$.

All samples, $\quad \mathrm{Co}(3 \quad \mathrm{~nm}) / \mathrm{NiO}(50 \quad \mathrm{~nm})$, $\mathrm{Co}(2 \mathrm{~nm}) / \mathrm{Ir}_{20} \mathrm{Mn}_{80}(20 \mathrm{~nm}) / \mathrm{Cu}(2.5 \mathrm{~nm}) / \mathrm{Ta}(5 \mathrm{~nm})$, and $\mathrm{Co}_{90} \mathrm{Fe}_{10}(\mathrm{t} \mathrm{nm}) / \operatorname{PtMn}(25 \mathrm{~nm}) / \mathrm{Ta}(5 \mathrm{~nm})$, with $\mathrm{t}=1 \mathrm{~nm}, 2 \mathrm{~nm}, 3 \mathrm{~nm}$, were grown at IBM by DC magnetron sputtering on $\mathrm{Si}$ wafers at room temperature under an Ar pressure of $2 \mathrm{~m}$ Torr. The $\mathrm{NiO}$ layer in particular was reactively sputtered from a $\mathrm{Ni}$ target under a partial oxygen pressure of $0.2 \mathrm{mTorr}$. The PtMn samples were annealed at $525 \mathrm{~K}$ for 4 hours in a field of 1 Tesla before depositing the ferromagnetic layer to introduce the antiferromagnetic order. To introduce the unidirectional anisotropy the $\mathrm{Co} / \mathrm{NiO}$ and $\mathrm{Co}_{90} \mathrm{Fe}_{10} / \mathrm{PtMn}$ samples were exchange biased by field annealing above their Néel temperature in 50 Oe. In contrast, one of the $\mathrm{Co} / \mathrm{Ir}_{20} \mathrm{Mn}_{80}$ samples was exchange biased by deposition in an applied magnetic field (field grown) while the second one was prepared without an applied field (zero-field grown) for reference purposes. All samples were protected with thin $(\sim 2 \mathrm{~nm})$ coatings $(\mathrm{Pd}, \mathrm{Pt}$ or $\mathrm{Ru})$ against oxidation. In all cases the remanent magnetization direction (easy axis) was in the plane of the sample.

X-ray absorption spectra were obtained by recording the sample current as a function of x-ray energy. We used $81 \%$ left and right elliptically polarized $\mathrm{x}$-rays from 


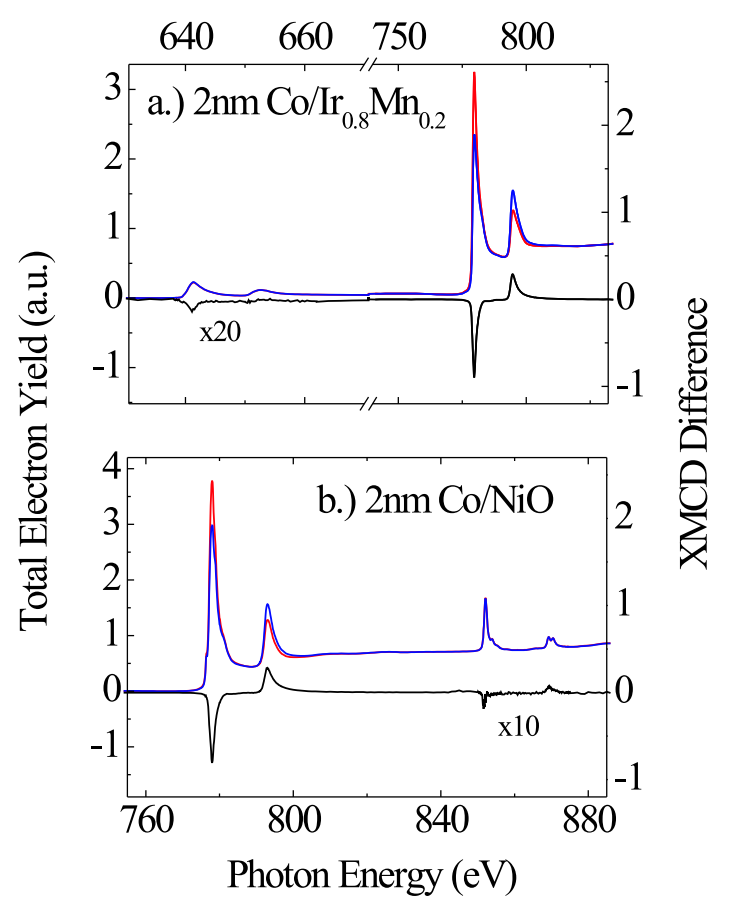

FIG. 1: XMCD spectra of (a) $\mathrm{Co}(2 \mathrm{~nm}) / \operatorname{Ir}_{20} \mathrm{Mn}_{80}(20 \mathrm{~nm})$ and $(b) \mathrm{Co}(3 \mathrm{~nm}) / \mathrm{NiO}(50 \mathrm{~nm})$. Shown are spectra for parallel (red) and antiparallel (blue) alignment of external field and xray helicity as well as their difference (black). Uncompensated and rotatable spins in the antiferromagnet are responsible for the observed Mn and Ni dichroism.

an elliptical polarized undulator on beamline 4.0.2 at the Advanced Light Source [14]. The x-rays were incident at a grazing angle of $30^{\circ}$ from the sample surface, either parallel or antiparallel to the bias direction. Magnetic fields of up to $\pm 0.28 \mathrm{~T}$ were applied along the $\mathrm{x}$-ray propagation direction using an electromagnet.

First, the total coverage with uncompensated spins at the interface is determined. Figs. 1a and b show absorption spectra measured on $\mathrm{Co} / \mathrm{IrMn}$ and $\mathrm{Co} / \mathrm{NiO}$, that were acquired for two opposite sample magnetization directions (red and blue) relative to the fixed helicity of the elliptically polarized x-rays. The difference spectra (below) demonstrate a magnetic circular dichroism at the Co $\mathrm{L}_{3}$ and $\mathrm{L}_{2}$ edges. In addition, uncompensated and rotatable $\mathrm{Ni}$ or $\mathrm{Mn}$ spins which follow the ferromagnetic Co spins near the surface of the antiferromagnet lead to a weak but still clearly visible ferromagnetic signal. For both systems it exhibits the same sign as the Co signal, demonstrating that the coupling between the magnetization of the FM and the AFM is parallel or ferromagnetic. From the size of the dichroism we estimate a nominal thickness of the rotatable magnetization of
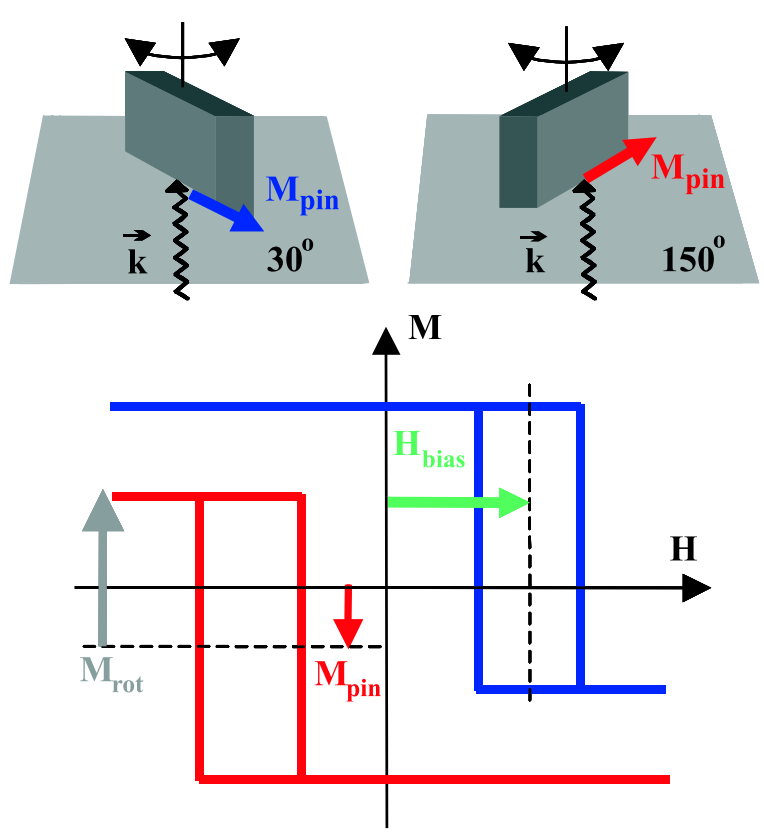

FIG. 2: Top: Experimental geometry used for the acquisition of the XMCD hysteresis loops. The $\vec{H}$ field is along the x-ray wavevector $\vec{k}$. The angle between exchange bias field and incident x-rays was varied between $30^{\circ}$ (blue) and $150^{\circ}$ (red). Bottom: Schematic hysteresis loops in the presence of rotating and pinned moments in an exchange biased sample. The arrows refer to the rotating magnetization (gray), the pinned magnetization (red) and the bias field (green).

$(0.44 \pm 0.11) \mathrm{ML}$ for $\mathrm{Co} / \mathrm{NiO}$ and $(0.52 \pm 0.13) \mathrm{ML}$ for $\mathrm{Co} / \mathrm{Ir}_{20} \mathrm{Mn}_{80}[22]$.

In order to determine whether all the observed interfacial spins are rotatable, element specific hysteresis loops $\vec{M}(\vec{H})$ were recorded. For clarity, the effect of a pinned magnetization onto the shape of the hysteresis loop is illustrated in Fig. 2. On top we show the two experimental geometries in which hysteresis loops were acquired, and the corresponding color-coded loops are shown below. The two loops describe a sample that contains rotating moments $\mathrm{M}_{\text {rot }}$ and a smaller number of pinned moments $\mathrm{M}_{\text {pin }}$. The height of each loop corresponds to twice the rotating magnetization $\mathrm{M}_{\text {rot }}$. The loops exhibit opposite horizontal and vertical shifts due to the reversal of the bias field direction relative to the applied field direction when the sample is rotated about a vertical axis. The vertical shift $M_{\text {pin }}$ is due to pinned moments that are not rotated by the applied field and therefore define the bias direction. From the asymmetry between vertical loop shift and loop height the ratio between pinned and rotating magnetization can be determined.

Four hysteresis loops were acquired by monitoring the sample current $I_{n}^{p}(\vec{H})$ as a function of the magnetic field $\vec{H}$ with the photon energy tuned to either of the $\mathrm{L}_{n}$ $(n=2,3)$ resonances and using left and right elliptically polarized $\mathrm{x}$-rays $(\mathrm{p}= \pm 0.81)$. The monitored cur- 


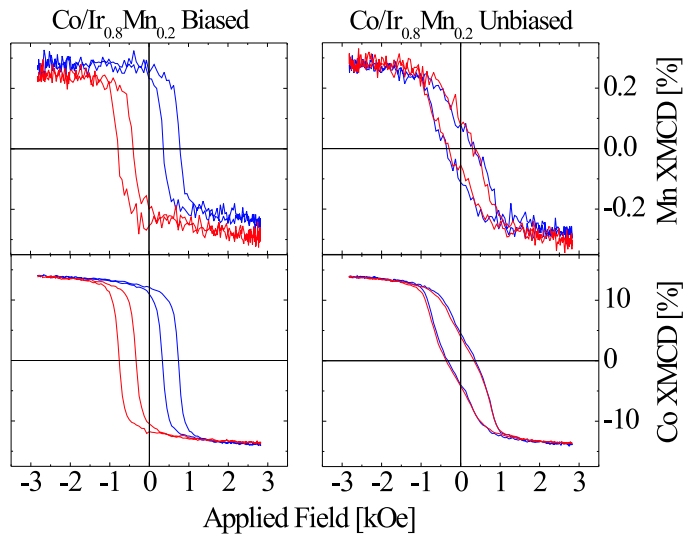

FIG. 3: Element specific XMCD hysteresis loops $\vec{M}(\vec{H})$ measured on Co and $\mathrm{Mn}$ in $\mathrm{Co}(2 \mathrm{~nm}) / \mathrm{Ir}_{20} \mathrm{Mn}_{80}(50 \mathrm{~nm})$. Loops of the field deposited sample are shown on the left while those from the zero-field deposited sample are shown on the right. The hysteresis loops were acquired with the bias field (horizontal loop shift) either parallel (red) or antiparallel (blue) to the propagation direction of the incident $\mathrm{x}$-rays.

rent for each loop is a superposition of the XMCD signal, non-magnetic background in the absorption signal and a modulation of the electron yield due to $\vec{H}$-field dependent deflection of the emitted electrons in the magnetic field. While the sample magnetization contributes with opposite sign to the asymmetry at the $\mathrm{L}_{3}$ and $\mathrm{L}_{2}$ edges, fielddependent artifacts contribute with the same sign. To cancel out these artifacts we used the four experimental loops to first calculate asymmetry ratios between loops obtained with opposite helicity of the x-rays ( $p= \pm 81 \%)$ at either the $\mathrm{L}_{3}$ or the $\mathrm{L}_{2}$ energy position and then took the difference of the ratios [23]. For the final results up to 20 loops were typically averaged with an acquisition time of 15 minutes per loop.

The experimental loops obtained from $\mathrm{Co}$ and $\mathrm{Mn}$ in field deposited (left) and zero-field deposited (right) $\mathrm{Co} / \mathrm{Ir}_{20} \mathrm{Mn}_{80}$ in the two different experimental geometries are shown in Fig. 3. We note upfront that corresponding data acquired on bare $\mathrm{Ir}_{20} \mathrm{Mn}_{80}$ films and normalized identically did not show any magnetic hysteresis and would simply yield a horizontal line at zero magnetization in Fig. 3. Also, because of the small size of the measured Mn signal we carefully checked the consistency of the measured shifts and normalization by an additional azimuthal rotation of the samples about the surface normal. The hysteresis loops acquired on the field deposited sample exhibit a rectangular shape and a horizontal shift of 570 Oersted. In contrast, the loops obtained from the zero-field deposited sample do not indicate the presence of a macroscopic uniaxial or unidirectional anisotropy. This is similar to the case of an exchange coupled sample, which has been ac-demagnetized during field cooling [12]. Here, due to the absence of a magnetic field during

\begin{tabular}{|c|c|c|c|c|}
\hline & Sample & $\rho(\mathrm{ML})$ & $\sigma_{\text {eff }}\left(\mathrm{mJ} / \mathrm{m}^{2}\right)$ & $\sigma\left(\mathrm{mJ} / \mathrm{m}^{2}\right)$ \\
\hline $\mathrm{A}$ & $3 \mathrm{~nm} \mathrm{Co} / \mathrm{NiO}$ & $0.04 \pm 0.01$ & $0.052 \pm 0.005$ & $1.3 \pm 0.5$ \\
\hline $\mathrm{B}$ & $2 \mathrm{~nm} \mathrm{Co} / \mathrm{IrMn}$ & $0.04 \pm 0.01$ & $0.168 \pm 0.020$ & $4.1 \pm 1.4$ \\
\hline $\mathrm{C}$ & $1 \mathrm{~nm} \mathrm{CoFe} / \mathrm{PtMn}$ & $0.03 \pm 0.01$ & $0.124 \pm 0.014$ & $3.9 \pm 1.4$ \\
\hline $\mathrm{D}$ & $2 \mathrm{~nm} \mathrm{CoFe} / \mathrm{PtMn}$ & $0.04 \pm 0.01$ & $0.188 \pm 0.015$ & $4.8 \pm 1.7$ \\
\hline $\mathrm{E}$ & $3 \mathrm{~nm} \mathrm{CoFe} / \mathrm{PtMn}$ & $0.04 \pm 0.01$ & $0.229 \pm 0.027$ & $5.7 \pm 2.0$ \\
\hline
\end{tabular}

TABLE I: Effective and corrected interface energies $\sigma$ calculated from the macroscopic loop shift and the coverage with pinned spins $\rho$.

growth, no orientation of the as-deposited antiferromagnetic $\operatorname{Ir}_{20} \mathrm{Mn}_{80}$ layer is achieved and hence an isotropic magnetic behavior is observed overall [13].

Both the Co spins in the FM and the Mn spins in the AFM exhibit identical coercivities and bias fields and also reveal an overall similar shape. In addition, the Mn loops of the field deposited sample exhibit a small vertical offset that is absent in the Mn loops of the zero-field deposited sample. The vertical offset in the Mn loops - as explained in Fig. 2 - indicates that in the biased sample a small fraction of about $(7 \pm 2) \%$ of the total uncompensated moments of $0.56 \pm 0.14 \mathrm{ML}$ is pinned, yielding a pinned coverage $\rho=(0.04 \pm 0.01) \mathrm{ML}$. On the other hand, the majority of uncompensated Mn moments rotates with the ferromagnetic Co. Similar results were obtained for $\mathrm{Co} / \mathrm{NiO}$ and $\mathrm{Co}_{90} \mathrm{Fe}_{10} / \mathrm{PtMn}$. The interface coupling in all three studied systems is parallel, and in all cases the thickness of the pinned magnetization was determined to lie in the range 0.03-0.04 monolayers (see table 1 ). No vertical shift was detected in any of the Co loops. Since the major part of the Co XMCD signal originates from the bulk of the film the absence of a vertical shift in the Co loop excludes the presence of pinned moments throughout the Co film. Even the same coverage with pinned Co spins at the interface as observed for IrMn would only lead to a vertical loop shift of less than $0.3 \%$, which is too small to be detected.

The amount of pinned interfacial magnetization is correlated to the observed macroscopic bias fields following the original idea of Meiklejohn and Bean[2, 3]. The macroscopic bias field $\vec{H}_{B}$ of an epitaxial, fully uncompensated and pinned ferromagnet/antiferromagnet interface can be written as a function of the unidirectional magnetic interface energy $\sigma$ and the Heisenberg-like interface exchange energy $J$, according to,

$$
H_{B}=\frac{\sigma}{M_{\mathrm{FM}} t_{\mathrm{FM}}}=J \frac{S_{\mathrm{AFM}} S_{\mathrm{FM}}}{a_{\mathrm{AFM}}^{2} M_{\mathrm{FM}} t_{\mathrm{FM}}}
$$

$M_{\mathrm{FM}}$, and $t_{\mathrm{FM}}$ are the magnetization and the thickness of the FM, a AFM is the size of the unit cell of the AFM, and $S_{\mathrm{FM}}$ and $S_{\mathrm{AFM}}$ are the atomic magnetic moments of the FM and the AFM. This model is applicable for artificial antiferromagnet/ferromagnet multilayer structures as shown by Jiang et al [15], yet fails in non-ideal 
systems, overestimating the interface coupling strength by orders of magnitude. Taking into account that only a small, pinned fraction $\rho$ of the uncompensated interfacial moments contributes to bias the ideal coupling energy $J$ and hence the interfacial energy density $\sigma$ can be substituted by effective values $J_{\text {eff }}$ and $\sigma_{\text {eff, which are defined }}$ in the following.

$$
\sigma_{\mathrm{eff}}=\rho \sigma, \quad J_{\mathrm{eff}}=\rho J
$$

The results for different materials and FM thicknesses are tabulated in Table 1. We list the nominal thickness $\rho$ of the pinned uncompensated layer in fractions of a monolayer and the effective interface energy $\sigma_{\text {eff }}$ calculated from the macroscopic exchange bias field. In agreement with numerous earlier studies $[4,5]$ we obtain laterally averaged interface coupling energies $\sigma_{\text {eff, }}$ which are about two orders of magnitude smaller than those expected for a fully uncompensated interface. This reduction is readily explained by the observed dilution of pinned uncompensated interface spins which can be used to renormalize the effective interface energy for the proper interface area leading to corrected interface energies $\sigma$ between $1 \mathrm{~mJ} / \mathrm{m}^{2}$ and $6 \mathrm{~mJ} / \mathrm{m}^{2}$. These values are close in size to the predicted interface energies of an ideal, uncompensated interface of about $10 \mathrm{~mJ} / \mathrm{m}^{2}$ [4, 5][24].

The values obtained for $\sigma$ for the three different PtMn samples (C, D and E) suggest that $\sigma$ increases with the thickness of the ferromagnetic layer. This behavior is observed because we assume in eqn. (1) that the exchange bias field is inversely proportional to the thickness of the ferromagnetic layer. Other studies have shown that this dependence is strict only above a certain minimum thickness of the ferromagnet (for a reference see [4]), which depends on the material and is typically a few nanometer. Below this thickness lower values for $H_{B}$ are observed as predicted by eqn. (1). Since the ferromagnetic films investigated here are close to the minimum thickness the results are affected by this deviation and therefore $\sigma$ is underestimated. Exchange biased samples with thicker ferromagnetic layers cannot be investigated in this setup because of the limited probing depth of the TEY approach.

In summary, we have observed pinned uncompensated spins at the interface of several exchange bias sandwiches that are used in room temperature device structures. In all cases we find that only $4 \%$ of the interface layer contains pinned spins. We believe that the tiny fraction of uncompensated pinned spins is the very reason that has impeded the unravelling of the exchange bias puzzle for nearly fifty years. We find that the size of the exchange bias field can be understood in terms of a simple extension of the early Meiklejohn and Bean model, which quantitatively explains the determined bias fields by the number of pinned interfacial moments and their size. It appears that the development of a domain wall parallel to the surface, either extending into the FM or the AFM [16-19] is not required, although its existence cannot be excluded. However, contributions to the unidirectional anisotropy energy from deeper layers in the antiferromagnet appear to be small relative to contributions originating from pinned spins in close proximity to the interface, which are detected by our method. The origin of the pinned spins still evades us, but it is tempting to speculate from their approximately constant number in the different samples (see Table I) and from the typical crystallographic grain size of $20 \mathrm{~nm}$ in our polycrystalline films [25] that they are located at grain (= domain) boundaries, since these cover about the same percentage of the interface.

* To whom correspondence should be addressed: hohldag@ stanford.edu

[1] B. Dieny et al., Phys. Rev. B 43, 1279 (1991).

[2] W. H. Meiklejohn and C. P. Bean, Phys. Rev. 102, 1413 (1956).

[3] W. H. Meiklejohn, J. Appl. Phys. 33, 1328 (1962).

[4] J. Nogués and I. K. Schuller, J. Magn. Magn. Mater. 192, 203 (1999).

[5] A. E. Berkowitz and K. Takano, J. Magn. Magn. Mater. 200, 552 (1999).

[6] K. Takano et al., Phys. Rev. Lett. 79, 1130 (1997).

[7] W. J. Antel, F. Perjeru, and G. R. Harp, Phys. Rev. Lett. 83, 1439 (1999).

[8] T. P. A. Hase et al., Appl. Phys. Lett. 79, 985 (2001).

[9] H. Ohldag et al., Phys. Rev. Lett. 87, 247201 (2001).

[10] J. Nogués, C. Leighton, and I. K. Schuller, Phys. Rev. B 61, 1315 (2000).

[11] T. Regan et al., Phys. Rev. B 64, 214422 (2001).

[12] N.J. Gökemeijer, J.W. Cai and C.L. Chien, Phys. Rev. B 60, 3033 (1999).

[13] F. Nolting et al., Nature 405, 767 (2000).

[14] A. T. Young et al., Nucl. Instr Meth. Res. A 467, 549 (2001).

[15] J. S. Jiang et al., Phys. Rev. B 61, 9653 (2000).

[16] M. D. Stiles and R. D. McMichael, Phys. Rev. B 59, 3722 (1999).

[17] D. Mauri, H. Siegmann, P. Bagus, and E. Kay, J. Appl. Phys. 62, 3047 (1987).

[18] M. Kiwi, J. Mejia-López, R. D. Portugal, and R. Ramírez, Appl. Phys. Lett. 75, 3995 (1999).

[19] P. Miltényi et al., Phys. Rev. Lett. 84, 4224 (2000).

[20] E. Goering et al., J. Appl. Phys. 88, 5920 (2000).

[21] M. Pajda et al., Phys. Rev. B 64, 174402 (2001).

[22] For this analysis we use the atomic moment of the ferromagnet and assume that all uncompensated spins are located at the interface. For IrMn we assume a magnetic moment of $3.4 \mu B$, for PtMn $4.2 \mu B$ and for Ni $2.0 \mu B$. The error bars result from the error with which the XMCD asymmetry can be determined $(10 \%)$ and the error which we allow for the magnetic moment (20\%).

[23] Altogether the resulting loop $\vec{M}(\vec{H})$ can be written as $\frac{I_{3}^{+}-I_{3}^{-}}{I_{3}^{+}+I_{3}^{-}}-\frac{I_{2}^{+}-I_{2}^{-}}{I_{2}^{+}+I_{2}^{-}}$, for a similar approach see Goering et al. $[20]$

[24] Furthermore, reasonable Heisenberg exchange constants 
of 1-7 meV[21] can be obtained by multiplying the corrected interface energy densities $\sigma$ with a typical antiferromagnetic unit cell area of $0.15 \mathrm{~nm}^{2}$

[25] The grain size of samples grown under the conditions described above has been determined by x-ray diffraction ( $\mathrm{IrMn} / \mathrm{PtMn})$ or atomic force microscopy $(\mathrm{NiO})$. 